\title{
Hadronic Light by Light Contributions to the Muon Anomalous Magnetic Moment With Physical Pions
}

\author{
Luchang Jin* \\ Physics Department, Columbia University, New York, New York 10027, USA \\ E-mail: 1 j2289@columbia.edu
}

\section{Thomas Blum}

Physics Department, University of Connecticut, Storrs, Connecticut 06269-3046, USA

RIKEN BNL Research Center, Brookhaven National Laboratory, Upton, New York 11973, USA

\section{Norman Christ}

Physics Department, Columbia University, New York, New York 10027, USA

\section{Masashi Hayakawa}

Department of Physics, Nagoya University, Nagoya 464-8602, Japan

Nishina Center, RIKEN, Wako, Saitama 351-0198, Japan

\section{Taku Izubuchi}

Physics Department, Brookhaven National Laboratory, Upton, New York 11973, USA

RIKEN BNL Research Center, Brookhaven National Laboratory, Upton, New York 11973, USA

\section{Christoph Lehner}

Physics Department, Brookhaven National Laboratory, Upton, New York 11973, USA

\begin{abstract}
The current measurement of muonic $g-2$ disagrees with the theoretical calculation by about 3 standard deviations. Hadronic vacuum polarization (HVP) and hadronic light by light (HLbL) are the two types of processes that contribute most to the theoretical uncertainty. The current value for HLbL is still given by models. I will describe results from a first-principles lattice calculation with a $139 \mathrm{MeV}$ pion in a box of $5.5 \mathrm{fm}$ extent. Our current numerical strategies, including noise reduction techniques, evaluating the $\mathrm{HLbL}$ amplitude at zero external momentum transfer, and important remaining challenges, in particular those associated with finite volume effects, will be discussed.
\end{abstract}

The 33rd International Symposium on Lattice Field Theory

14 - 18 July 2015

Kobe International Conference Center, Kobe, Japan

\footnotetext{
* Speaker.
} 


\section{Introduction}

The anomalous magnetic moment of muon, $a_{\mu}$, can be defined in terms of the photon-muon vertex function:

$$
\left\langle\mathbf{p}^{\prime}, s^{\prime}\left|j_{v}\left(\mathbf{x}_{\mathrm{op}}=0\right)\right| \mathbf{p}, s\right\rangle=-e \bar{u}_{s^{\prime}}\left(\mathbf{p}^{\prime}\right)\left[F_{1}\left(q^{2}\right) \gamma_{v}+i \frac{F_{2}\left(q^{2}\right)}{4 m}\left[\gamma_{v}, \gamma_{\rho}\right] q_{\rho}\right] u_{s}(\mathbf{p}),
$$

where $F_{1}\left(q^{2}=0\right)=1, F_{2}\left(q^{2}=0\right)=\left(g_{\mu}-2\right) / 2 \equiv a_{\mu}, q=p^{\prime}-p$. The formula is written in a Euclidean gamma matrix convention, $\left[\gamma_{\mu}, \gamma_{v}\right]=\delta_{\mu, v}$. Its value has been measured very precisely by BNL E821 [3]. It can also be calculated theoretically to great precision [8]. The three standard deviation $(287 \pm 80) \times 10^{-11}$ difference between experiment and theory makes muon $g-2$ a very interesting quantity. Much more accurate experiments, Fermilab E989 [11] and J-PARC E34 [14], are expected to start in a few years, so a more accurate theoretical determination will be necessary. Figure 1 shows the two diagrams that are the major sources of the theoretical uncertainty.

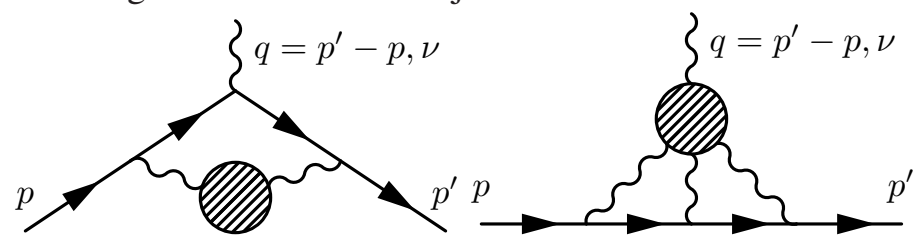

Figure 1: (Left) Hadronic vacuum polarization (HVP). (Right) Hadronic light-by-light (HLbL).

In this paper, we will only discuss the lattice calculation of connected hadronic light-by-light amplitude. Previously this quantity has only been calculated using models [15]. Attempts using lattice QCD were begun by T. Blum, M. Hayakawa, and T. Izubuchi more than 5 years ago [12, 6]. We have improved the methodology dramatically, as described in Ref [13, 7], which leads to a reduction in statistical errors by more than an order of magnitude. Since much of the material that was presented at LATTICE 2015 has now appeared in a long paper [7], this proceedings is devoted to an expanded discussion of three topics that were only briefly presented during the conference: a) The sampling strategy that concentrated the result on the more easily evaluated, short distance region in position space. b) A new proposal to perform the QCD and QED portions of the HLbL calculation in different space-time volumes and c) First numerical results from a physical-quarkmass, $48^{3} \times 96$ study.

\section{Sampling Strategy}

We start the discussion by repeating our final formula for evaluating the light-by-light contribution to $F_{2}\left(q^{2}=0\right)$ obtained in $\operatorname{Refs}[13,7]$.

$$
\frac{F_{2}\left(q^{2}=0\right)}{m} \frac{\left(\sigma_{s^{\prime} s}\right)_{i}}{2}=\sum_{r, \tilde{z}} \mathfrak{Z}\left(\frac{r}{2},-\frac{r}{2}, \tilde{z}\right) \sum_{\tilde{x}_{\mathrm{op}}} \frac{1}{2} \varepsilon_{i j k}\left(\tilde{x}_{\mathrm{op}}\right)_{j} \cdot i \bar{u}_{s^{\prime}}(\overrightarrow{0}) \mathscr{F}_{k}^{C}\left(\frac{r}{2},-\frac{r}{2}, \tilde{z}, \tilde{x}_{\mathrm{op}}\right) u_{s}(\overrightarrow{0}) .
$$

where $\left(\sigma_{s^{\prime} s}\right)_{i}=\bar{u}_{s^{\prime}}(\overrightarrow{0}) \Sigma_{i} u_{s}(\overrightarrow{0})$ are the conventional Pauli matrices, $\Sigma_{k}=\frac{1}{4 i} \varepsilon_{i j k}\left[\gamma_{i}, \gamma_{j}\right]$ and the weight function " $\mathfrak{Z}$ " is defined below but could be replaced by 1 . The integration variables are related to the coordinates in Figure 2 by the following equations: $r=x-y, \tilde{z}=z-(x+y) / 2, \tilde{x}_{\mathrm{op}}=$ $x_{\mathrm{op}}-(x+y) / 2$, while

$$
\mathscr{F}_{v}^{C}\left(x, y, z, x_{\mathrm{op}}\right)=\frac{1}{3} \mathscr{F}_{v}\left(x, y, z, x_{\mathrm{op}}\right)+\frac{1}{3} \mathscr{F}_{v}\left(y, z, x, x_{\mathrm{op}}\right)+\frac{1}{3} \mathscr{F}_{v}\left(z, x, y, x_{\mathrm{op}}\right),
$$




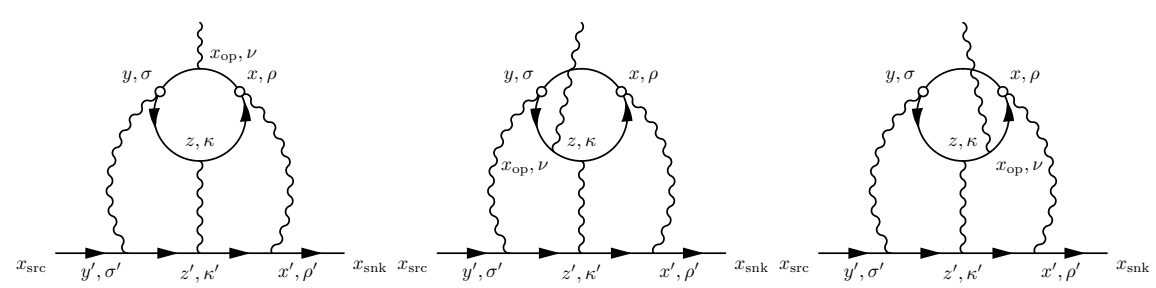

Figure 2: Diagrams showing the three different ways of inserting the external photon when the vertices $x$ and $y$ are fixed. For each of these three diagrams there are five other possible permutations of the connections between the three internal photons and the muon line that are not shown.

The amplitude $\mathscr{F}_{v}\left(x, y, z, x_{\mathrm{op}}\right)$ is given by:

$$
\begin{aligned}
& \mathscr{F}_{v}\left(x, y, z, x_{\mathrm{op}}\right) \\
= & \left\langle-\sum_{q=u, d, s}\left(e_{q} / e\right)^{4} \operatorname{tr}\left[\gamma_{\rho} S_{q}(x, z) \gamma_{\kappa} S_{q}(z, y) \gamma_{\sigma} S_{q}\left(y, x_{\mathrm{op}}\right) \gamma_{v} S_{q}\left(x_{\mathrm{op}}, x\right)\right]\right\rangle_{\mathrm{QCD}} \\
\cdot & \sum_{x^{\prime}, y^{\prime}, z^{\prime}}(-i e)^{6} G_{\rho \rho^{\prime}}\left(x, x^{\prime}\right) G_{\sigma \sigma^{\prime}}\left(y, y^{\prime}\right) G_{\kappa \kappa^{\prime}}\left(z, z^{\prime}\right) \\
\cdot & e^{m_{\mu}\left(t_{\mathrm{snk}}-t_{\mathrm{src}}\right)} \sum_{\vec{x}_{\mathrm{snk}}, \vec{x}_{\mathrm{src}}}\left[S_{\mu}\left(x_{\mathrm{snk}}, x^{\prime}\right) \gamma_{\rho^{\prime}} S_{\mu}\left(x^{\prime}, z^{\prime}\right) \gamma_{\kappa^{\prime}} S_{\mu}\left(z^{\prime}, y^{\prime}\right) \gamma_{\sigma^{\prime}} S_{\mu}\left(y^{\prime}, x_{\mathrm{src}}\right)\right. \\
\cdot & +S_{\mu}\left(x_{\mathrm{snk}}, z^{\prime}\right) \gamma_{\kappa^{\prime}} S_{\mu}\left(z^{\prime}, x^{\prime}\right) \gamma_{\rho^{\prime}} S_{\mu}\left(x^{\prime}, y^{\prime}\right) \gamma_{\sigma^{\prime}} S_{\mu}\left(y^{\prime}, x_{\mathrm{src}}\right)+\text { other 4 permutations], }
\end{aligned}
$$

where $e_{u} / e=2 / 3, e_{d} / e=e_{s} / e=-1 / 3$.

We perform the sum over $r=x-y$ by sampling a few values on each QCD configuration and use point source propagators at $x$ and $y$. The sum over $\tilde{x}_{\mathrm{op}}$ can be performed as a sequential source, $\tilde{z}$ is summed over as a sink. Since $x$ and $y$ are connected by quark lines, it can be expected that the major contribution to $g-2$ comes from the region where $r$ is small. We accommodate this by using importance sampling, that is we sample the small $r$ region more often, then divide the sample result by the probability. We even perform a complete sum, up to discrete symmetries, in the region where $r \leqslant r_{\max } . r_{\max }$ is usually chosen to be 5 in lattice units in our numerical simulations.

In our primary calculation we choose $\mathfrak{Z}(x, y, z)$ to be given by

$$
\mathfrak{Z}(x, y, z)= \begin{cases}3 & \text { if }|x-y|<|x-z| \text { and }|x-y|<|y-z| \\ 3 / 2 & \text { if }|x-y|=|x-z|<|y-z| \text { or }|x-y|=|y-z|<|x-z| \\ 1 & \text { if }|x-y|=|x-z|=|y-z| \\ 0 & \text { otherwise }\end{cases}
$$

This choice of $\mathfrak{Z}$ restricts the summation region for $z$, in such a way as to suppress the contribution from the large $r$ region. This way, the connected $\pi^{0}$ exchange contribution would be completely captured by the small $r$ region if we sum up to the size of the pion, instead of the Compton wave length of the pion. We may also try the opposite of this choice, which can provide more information about QCD finite-volume effects, and the size of the long-distance $\pi^{0}$-exchange contribtion to the light-by-light process:

$$
\mathfrak{Z}^{\prime}(x, y, z)= \begin{cases}3 & \text { if }|x-y|>|x-z| \text { and }|x-y|>|y-z| \\ 3 / 2 & \text { if }|x-y|=|x-z|>|y-z| \text { or }|x-y|=|y-z|>|x-z| \\ 1 & \text { if }|x-y|=|x-z|=|y-z| \\ 0 & \text { otherwise }\end{cases}
$$


With this choice, in the large $r$ region, most of the contribution should come from the connected $\pi^{0}$ exchange. How rapidly this contribution decays could give us a hint about the size of the QCD finite-volume effect, which comes from the quark propagators evaluated within the finite size lattice.

\section{QCD Box Inside a Larger QED Box}

The QCD finite volume effects are exponentially suppressed in the linear size of the lattice volume times $m_{\pi}$, the energy of lowest energy eigen-state of QCD. There are also QED finite volume effects, which are caused by the photon and the muon propagators being evaluated within that finite volume, and the summations over $\vec{x}_{\text {src }}, \vec{x}_{\mathrm{snk}}, x^{\prime}, y^{\prime}, z^{\prime}$ being controlled to lie within that finite volume. Because the photon is massless, the QED finite volume effects are power like, similar to many other lattice calculations involving QED. In this particular case, these QED finite volume effects scale like $1 / L^{2}$ as discussed in Ref [13,7]. These QED finite volume effects can be reduced by evaluating the photon and muon propagators and performing the summation over $\vec{x}_{\text {src }}, \vec{x}_{\text {snk }}, x^{\prime}$, $y^{\prime}, z^{\prime}$ in Eq. (2.3) in a larger volume compared to that in which the QCD part of the calculation is performed. We refer to the former as the QED box and the latter as the QCD box, see Figure 3. With our current light-by-light evaluation strategy, the computations for the quark and the muon propagators are almost independent. We can compute the light-by-light process for a few different QED boxes without recomputing the quark part. One can then extrapolate to infinite volume based on these results from different QED boxes. Since the quark part is the same, we expect there will exist strong correlations between these results, which would benefit the extrapolation. In principle, one could evaluate the muon and photon propagators using the continuum formulae and perform the coordinate-space QED summation in infinite volume directly, thus completely eliminating this $\mathscr{O}\left(1 / L^{2}\right)$ finite volume effect. In fact, this is exactly the strategy for the HVP calculation, where the usual approach [4] can be viewed as substituting the finite-volume result for $\Pi\left(q^{2}\right)$ into one- or two-loop QED calculations performed in infinite volume. At this point, one can see that computing the QED part of the diagram in a larger, possibly infinite, QED box is a quite general idea, and could be applied in many (but not necessarily all) other lattice QCD calculations involving QED. In some cases, like the QED mass-splittings, all one may need to evaluate is the photon propagator in infinite volume. Christoph Lehner talks about this in greater details in his talk at LATTICE 2015.

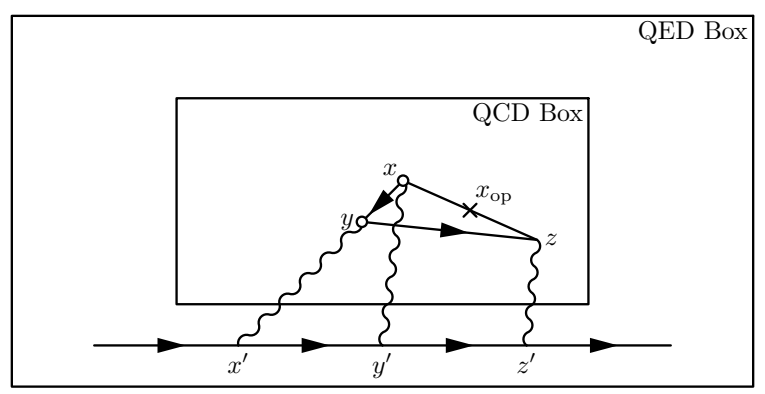

Figure 3: QCD box inside QED box illustration.

As discussed above, the finite volume effects of the light-by-light calculation come from two sources. One source is the quark loop is evaluated using a finite QCD box, which is exponentially suppressed in the size of the QCD box. The other source is the photon and muon propagators 
evaluated in the QED box, which scales like $1 / L^{2}$ as discussed in Ref [13, 7]. Here, we the strategy of making the QED box larger than QCD box by a calculation in $16^{3}$ and $24^{3}$ lattice volumes $[1,2]$ but with the same lattice spacing and pion mass. All computations are performed on 14 configurations separated by $200 \mathrm{MD}$ time unit and the results are shown in Table 1.

$\begin{array}{ccccc}\text { Ensemble } & \text { QCD Size } & \text { QED Size } & t_{\text {snk }}-t_{\text {src }} & \frac{F_{2}\left(q^{2}=0\right)}{(\alpha / \pi)^{3}} \\ \text { 16I [1] } & 16^{3} \times 32 & 16^{3} \times 32 & 16 & 0.1158(8) \\ \text { 24I [2] } & 24^{3} \times 64 & 24^{3} \times 64 & 32 & 0.2144(27) \\ \text { 16I-24 [1] } & 16^{3} \times 32 & 24^{3} \times 64 & 32 & 0.1674(22)\end{array}$

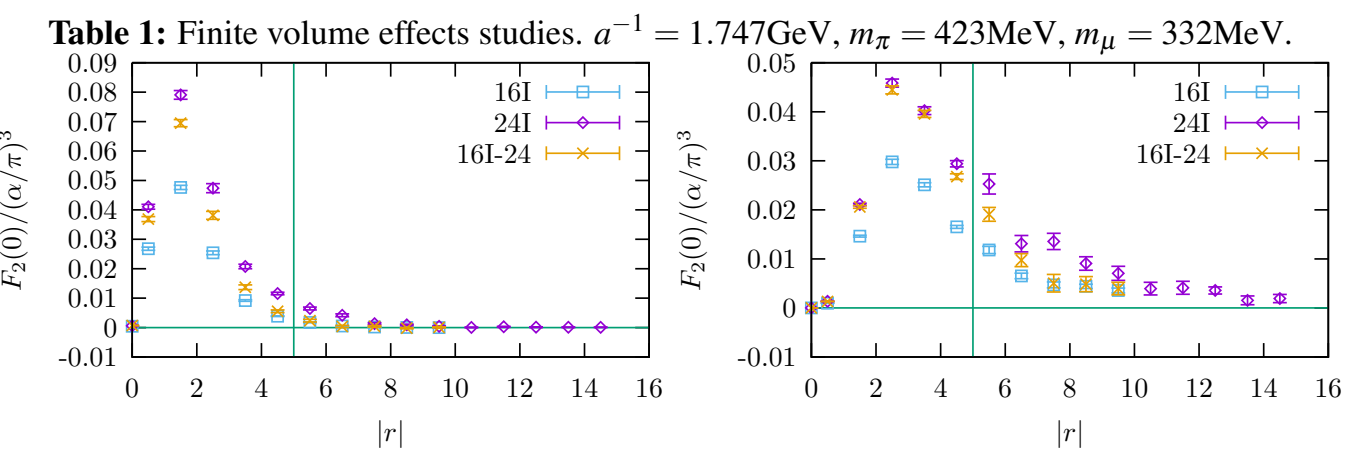

Figure 4: Above plots show histograms of the contribution to $F_{2}$ from different separations $|r|=|x-y|$. The sum of all these points gives the final result for $F_{2}$. The vertical lines at $|r|=5$ in the plots indicate the value of $r_{\text {max }}$. The left plot is evaluated with $\mathfrak{Z}$, so the small $r$ region includes most of the contribution. The right plot is evaluated with $\mathfrak{Z}^{\prime}$ in place of $\mathfrak{Z}$, so the QCD finite volume is better controlled in the small $r$ region.

We can see that by using only a larger QED lattice, the major part of the finite-volume effects have been removed. However the disagreement between the results shown in the 2nd and 3rd lines of Table 1 implies that a $16^{3}$ lattice with a spatial extent of $1.8 \mathrm{fm}$ is not large enough to entirely suppress the QCD finite volume effect. This can be seen from the right plot of Figure 4. In the small $r$ region, where we control the QCD finite volume effects. the result from the 16I QCD/24 QED calculation agrees very well with 24I. However, as $|r|$ becomes larger, the quark loop evaluated in $16 \mathrm{I}$ is affected by the boundary and begins to deviate from the 24I results. Note because we use periodic boundary conditions for the quark propagators, the maximum spatial separation between source and sink in any direction is 8 for quark propagators on the $16 \mathrm{I}$ lattice.

\section{Physical Pion Mass $48^{3}$ Lattice Simulation}

Here we report on our ongoing numerical study, being done in a larger collaboration, on a $48^{3}$ lattice with a physical pion mass, and a spatial extent of 5.5fm [5]. The computation has so far been completed on 30 configurations separated by $40 \mathrm{MD}$ time units. The AMA [9] technique is used to reduce the statistical error. The small AMA correction term is given in Table 2 with the label AMA, and has already been added to the total contribution listed in the table. We also plot the histograms in Figure 5, which just contain results from the imprecise solves.

$\begin{array}{cccccc}\text { Ensemble } & \text { QCD Size } & \text { QED Size } & t_{\text {snk }}-t_{\text {src }} & \frac{F_{2}\left(q^{2}=0\right)}{(\alpha / \pi)^{3}} & \text { AMA } \\ \text { 48I [5] } & 48^{3} \times 96 & 48^{3} \times 96 & 40 & 0.0926(124) & 0.0008(23) \\ \text { 48I [5] } & 48^{3} \times 96 & 48^{3} \times 96 & 48 & 0.0946(131) & 0.0007(24)\end{array}$

Table 2: Finite volume effects studies. $a^{-1}=1.73 \mathrm{GeV}, m_{\pi}=139 \mathrm{MeV}, m_{\mu}=106 \mathrm{MeV}$. 
The results for the two different time separations are quite close suggesting that the effect of excited states is under control. Based on our finite volume and finite lattice spacing in our pure QED simulations in Ref [13,7], we would estimate the above result could have $20 \%$ discretization errors and significant finite volume errors. As a result, the infinite volume, continuum value for the connected light-by-light contribution could be twice as large as this value. However, the disconnected light-by-light diagrams may contribute negatively and cancel part of the above enhancement.
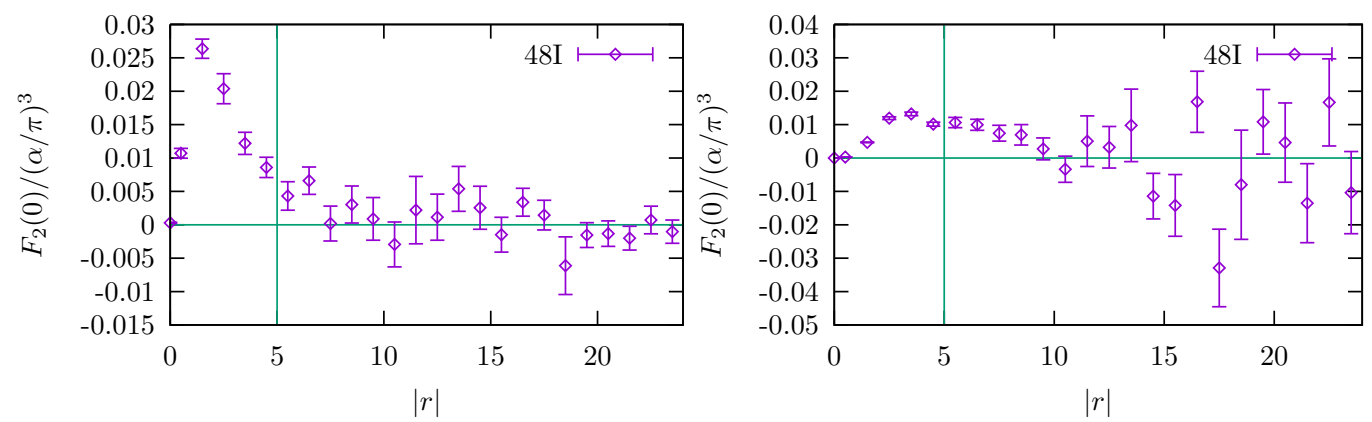

Figure 5: Histograms of the contribution to $F_{2}$ from different separations $|r|=|x-y|$. The sum of all these points gives the final result for $F_{2}$. The vertical lines at $|r|=5$ indicate the value of $r_{\max }$. The left plot is evaluated with $\mathfrak{Z}$, so the small $r$ region includes most of the contribution. The right plot is evaluated with $\mathfrak{Z}^{\prime}$ in place of $\mathfrak{Z}$, so the QCD finite volume effects are better controlled in the small $r$ region. Currently we only have 8 configurations to make the plot on the right.

The plot on the right of Figure 5 suggest that the QCD finite volume effects will likely to be small, because it seems that the contribution vanishes rather quickly at $|r| \sim 10$, much smaller than 24 , which is half of the spatial size of the lattice. However, more statistics are needed to draw a firm conclusion.

\section{Conclusions and Acknowledgments}

We have described two sampling strategies for our choice of stochastic integration points. We have also presented a new lattice-based numerical method for reducing the QED power-law, finite volume error by introducing a second, larger "QED volume" in which the photon and muon parts of the path integral are evaluated. This method has been illustrated by comparing HLbL calculated on combinations of $16^{3}, 24^{3}$ volumes. Finally we have shown our current result using this method on a physical-pion-mass, $48^{3}, 5.5 \mathrm{fm}$ lattice. We plan to a) address the discretization errors by computing on our finer, physical-pion $64^{3}$ lattice with similar physical volume. b) address the finite volume effect by using the $48^{3}$ QCD box inside a larger QED box and c) compute disconnected diagrams within the framework of this newly-developed evaluation strategy.

We would like to thank our RBC and UKQCD collaborators for helpful discussions and support. We would also like to thank RBRC for BG/Q computer time. The $48^{3}$ computation is performed on Mira with ALCC allocation using BAGEL [10] library. T.B is supported by U.S. DOE grant \#DE-FG02-92ER41989. N.H.C and L.C.J are supported by U.S. DOE grant \#DESC0011941. M.H is supported by Grants-in-Aid for Scientific Research \#25610053. T.I and C.L are supported by U.S. DOE Contract \#AC-02-98CH10996(BNL). T.I is also supported by Grantsin-Aid for Scientific Research \#26400261. This research used resources of the Argonne Leadership Computing Facility, which is a DOE Office of Science User Facility supported under Contract DEAC02-06CH11357. 


\section{References}

[1] C. Allton et al. 2+1 flavor domain wall QCD on a (2 fm)*83 lattice: Light meson spectroscopy with L(s) = 16. Phys.Rev., D76:014504, 2007, hep-lat/0701013.

[2] C. Allton et al. Physical Results from 2+1 Flavor Domain Wall QCD and SU(2) Chiral Perturbation Theory. Phys.Rev., D78:114509, 2008, 0804.0473.

[3] G.W. Bennett et al. Final Report of the Muon E821 Anomalous Magnetic Moment Measurement at BNL. Phys.Rev., D73:072003, 2006, hep-ex/0602035.

[4] T. Blum. Lattice calculation of the lowest order hadronic contribution to the muon anomalous magnetic moment. Phys. Rev. Lett., 91:052001, 2003, hep-lat/0212018.

[5] T. Blum et al. Domain wall QCD with physical quark masses. 2014, 1411.7017.

[6] Thomas Blum, Saumitra Chowdhury, Masashi Hayakawa, and Taku Izubuchi. Hadronic light-by-light scattering contribution to the muon anomalous magnetic moment from lattice QCD. Phys.Rev.Lett., 114(1):012001, 2015, 1407.2923.

[7] Thomas Blum, Norman Christ, Masashi Hayakawa, Taku Izubuchi, Luchang Jin, and Christoph Lehner. Lattice Calculation of Hadronic Light-by-Light Contribution to the Muon Anomalous Magnetic Moment. 2015, 1510.07100.

[8] Thomas Blum, Achim Denig, Ivan Logashenko, Eduardo de Rafael, B. Lee Roberts, et al. The Muon (g-2) Theory Value: Present and Future. 2013, 1311.2198.

[9] Thomas Blum, Taku Izubuchi, and Eigo Shintani. New class of variance-reduction techniques using lattice symmetries. Phys. Rev., D88(9):094503, 2013, 1208.4349.

[10] Peter A. Boyle. The BAGEL assembler generation library. Comput. Phys. Commun., 180:2739-2748, 2009.

[11] Frederick Gray. Muon g-2 Experiment at Fermilab. In 12th Conference on the Intersections of Particle and Nuclear Physics (CIPANP 2015) Vail, Colorado, USA, May 19-24, 2015, 2015, 1510.00346.

[12] Masashi Hayakawa, Thomas Blum, Taku Izubuchi, and Norikazu Yamada. Hadronic light-by-light scattering contribution to the muon g-2 from lattice QCD: Methodology. PoS, LAT2005:353, 2006, hep-lat/0509016.

[13] Luchang Jin, Thomas Blum, Norman Christ, Masashi Hayakawa, Taku Izubuchi, and Christoph Lehner. Lattice Calculation of the Connected Hadronic Light-by-Light Contribution to the Muon Anomalous Magnetic Moment. In 12th Conference on the Intersections of Particle and Nuclear Physics (CIPANP 2015) Vail, Colorado, USA, May 19-24, 2015, 2015, 1509.08372.

[14] Shoichiro Nishimura et al. Design of the Positron Tracking Detector for the Muon g - 2/EDM Experiment at J-PARC. JPS Conf. Proc., 8:025015, 2015.

[15] Joaquim Prades, Eduardo de Rafael, and Arkady Vainshtein. Hadronic Light-by-Light Scattering Contribution to the Muon Anomalous Magnetic Moment. 2009, 0901.0306. 